\title{
The Application of W. Kent Muhlbauer's Model For The Risk Assessment of District Heating Networks
}

\author{
Malgorzata Kwestarz, Ph.D. in Engineering \\ Warsaw University of Technology, Building Services, Hydro and Environmental Engineering Department, \\ District Heating and Gas Systems Division, 00-653 Warsaw, Nowowiejska Street 20, Poland
}

\begin{abstract}
This scientific paper explains the effectiveness of W.K. Muhlbauer's model in district heating networks. Its major focus is upon determining the level of risk of failure for heating network and as well as seeking a compromise between the capital expenses and the level of occupational safety.The use of software SimNet SSV Heat in our study case, efficiently determines the level of risk associated with leakage and predicts the behavior of leakage within pipeline of any length. It also reduces the level of risk normally associated with additional costs at the designing stage, construction and operations.
\end{abstract}

Keywords: risk analyses, risk management, district heating network.

\section{Introduction}

Risk management is defined as taking an action, whose aim is to identify, assess and control risks and complete control of the action taken. The main objective of risk management, in the case of pipeline systems, is to reduce the occurrence of a failure or when it occurs to limit its effects. Network systems may have potential dangers for network users and as well as over the surrounding environment $[1,4,8]$.

Evaluation of operational reliability is most often referred to the problem of determining the frequency of failure and its causes. Reliability by analogy to other systems can be defined as follows: "The reliability of the piping system is a function of the fulfillment of the task delivery of the specified medium in the required quantity and quality".

The direct relation to security could be:

- adverse events,

- threats

- losses

Adverse events are identified with a failure of system security that poses a threat to protected goods.As a measure of safety, the level of risk is used that determines the likelihood of a hazard and associated losses.

Pipeline systems damage cannot be completely eliminated. These are extensive systems consisting of many separate subsystems operating in different changing conditions. The risk of failure is a common phenomenon, but the determination of this risk and its management until it decreases to a considerable extent the frequency of the failure and reduce effects of their occurrence [3].

Risk Management is understood as taking activities aimed at:

- Recognition;

- Evaluation;

- Risk control;

- Control of actions taken.

The purpose of management is to reduce the risk and protect against its effects. The purpose of diagnosis is to identify the risks that are associated with the investment under consideration. Their proper diagnosis is important in that it allows the investor to take action to protect or reduce them.

Risk Assessment is carried out using a variety of measures. Their choice depends on the type of risk to be assessed. Using quantification, it is possible to identify these risk factors and special attention should be paid to them.

Controlling is understood as an action to be taken to minimize the risk to the acceptable level. There are two main approaches to risk control:

- Active which is based on the impact over the causes of the risk;

- Passive focusing upon prevention against possible losses. 


\section{Risk Assessment}

The risk assessment (giving it a probabilistic measure) is an element of the risk management process which is preceded by a risk analysis, i.e. the action of acquiring available information about the hazards of the underlying system to take risks. Risk is a function [1]:

- Variables characterizing the probability of loss;

- Variables characterizing the size of the loss.

Relationship between risk metrics and uncertainty measures presents the general equation:

$$
(\text { Level of Risk })=(\text { Level of Failure }) x(\text { Level of Hazard })(1)
$$

The level of failure is usually expressed by the probability of occurrence of an adverse event, while the level of threat is expressed by expected losses, ie the effects of this event.

Risk $=P(Z) x S(Z)$

Where;

$P(Z)$ - the probability of occurrence of adverse events $Z$

$S(Z)$ - effects (loss) caused by the occurrence of an event $Z$.

From the relation (2) we see that the risk can be reduced by reducing the probability of occurrence of adverse events - or by reducing the effects of this event, if at all happens.

Risk analysis can be carried out - either by the quantitative method or by the qualitative method. Quantitative methods allow for a more rational evaluation, comparison and action for safety as compared to qualitative methods. However, they are much more difficult to use and require a long set of input information. For quantitative risk analysis, it is necessary to have a suitable model - a probabilistic risk model that should consist of:

- hazard model, which allows the determination of hazard measures;

- a model of reliability that makes it possible to designate appropriate - the probability of adverse events, including probability of primary dangerous events;

- relationship between these models.

Qualitative methods are part of coarse risk assessment methods. Qualitative risk assessment is most often a subjective assessment based on good practice and experience. The result of this estimation is the list of threats together with a relative risk ranking. In the qualitative methods, the risk and potential consequences of its occurrence are presented descriptively. An example of a qualitative risk assessment method is Mühlbauer`s method [4]. It can be used successfully to estimate risk in situations where the lack of input information does not permit the construction of an accurate probabilistic model.

It is a point system belonging to the so-called subjective methods for estimating risks.

The numerical values are assigned to the parameters that affect the risk associated with the operation of pipeline system exploration.

Advantages of this approach: - As our knowledge and experience are growing, the risk assessment algorithm can be relatively easily modified.

\section{The Basics of WKM's Model Risk Assessment}

Basis for assessing the level of risk according to WKM is the division of the assessed pipeline into sections[4,5]. The more are the sections - more detailed is the analysis. However, too much fragmentation can lead to an overwhelming number of results, which in turn distorts the picture of real threats.

Decomposition of pipeline into sections assumes that evaluated part of the network has the fixed geometrical dimensions.

Each section of the pipeline is rated by an expert. In the case of risk analysis, the term "expert" means a team of people who possess the necessary knowledge, qualifications, experience and knowledge of the analyzed network. In practice, it is a team composed of the designer, the investment contractor and a person responsible for the current operation of the network traffic department, who assess the network within their competence. Such a complex team reduces the impact of subjective judgment, which allows the method to be considered objective.

Ratings are made in four groups of factors called indicators [4,5]:

- Indicator of damage by a third party;

- Indicator of corrosion;

- Indicator of rate design;

- Indicator of invalid operations. 
The order of indicators does not matter, because each of them can describe a selected section of the pipeline on a scale of 0 to 100 points.Upon completion of the assessment of the above indicators, the number of points awarded is aggregated and multiplied by the leakage rate index. The latter leakage rate indicator is the weight that terminates the risk analysis for the selected network segment. The indicator takes into account the hazards of the product being transported to the environment, including the environment and the intensity of urbanization surrounding the pipeline. The leakage rate is important because it takes into account the risk to human life.

Point scoring allows you to analyze the risk within the subject network. It types the sections of the network structure analyzed which have the smallest number of points allocated, which translates into the highest level of risk, but is related to the remaining sections of a particular network. The WKM method is a qualitative method despite point analysis. The resulting numerical result is not the absolute value of the risk level but relative to the other parts of the same network.

The relative risk score may range from 0 points (theoretically) to 400 points as the sum of the I, II, III and IV ratios multiplied by the efflux coefficient which can range from 0.2 to 88 .

The higher the number of points, the higher the risk of network segment exploitation.

\section{Extended Risk Assessment of WKM's Model}

The basic risk assessment model can be expanded with additional indicators that are relevant in certain situations. It means that they may occur in selected network analysis system.

Figure 1 shows a diagram of indicators in the extended method of W. Kent a Muhlbauer.

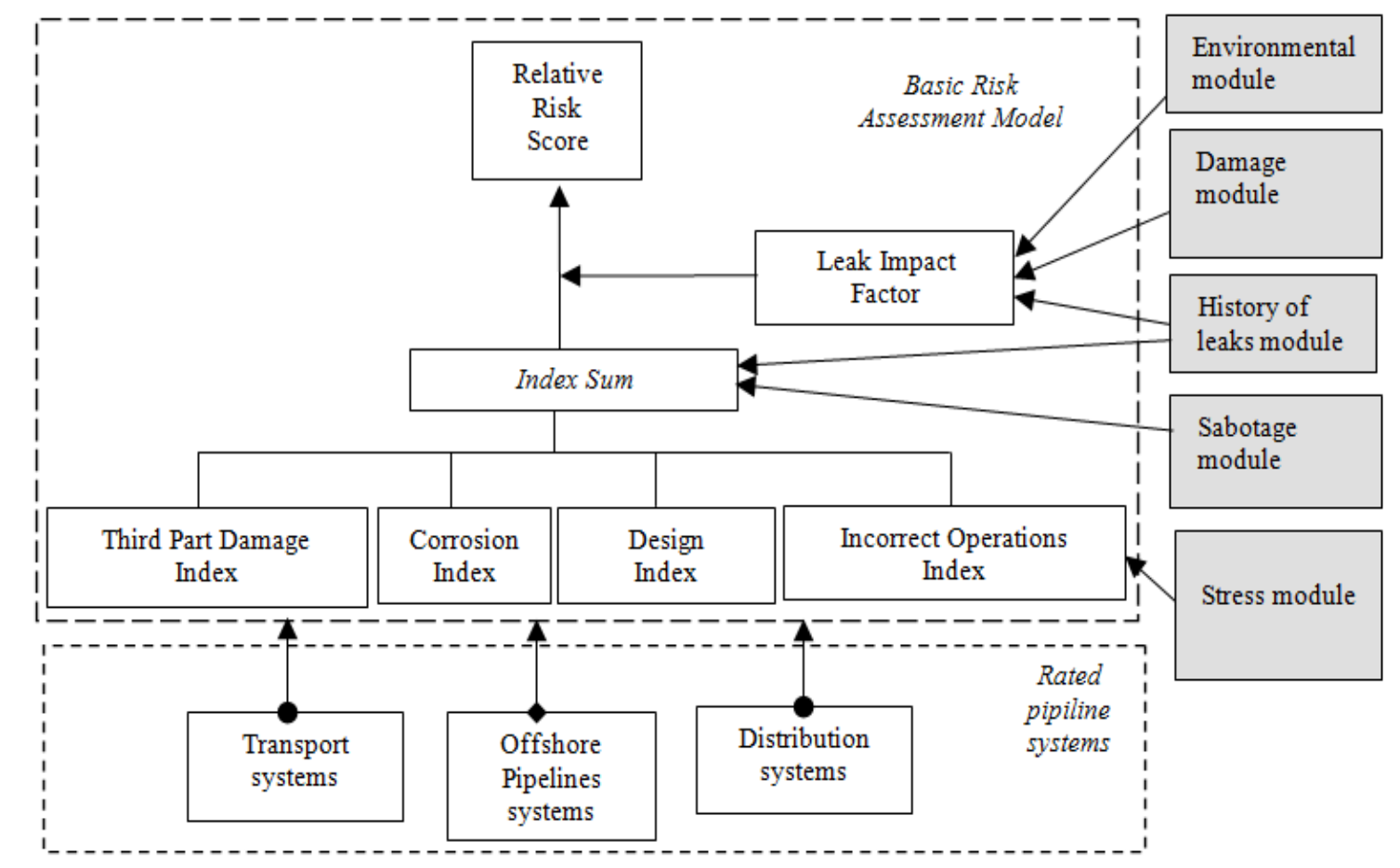

Fig.1 Diagram of WKM enhanced risk assessment method [4]

Extended method optionally includes five additional modules added to the basic risk assessment method:

- Environmental module;

- Damage module;

- History of leaks module;

- $\quad$ Sabotage module;

- $\quad$ Stress module. 


\subsection{Environmental Module}

The diagram of the environmental module interaction is shown in Figure 2.

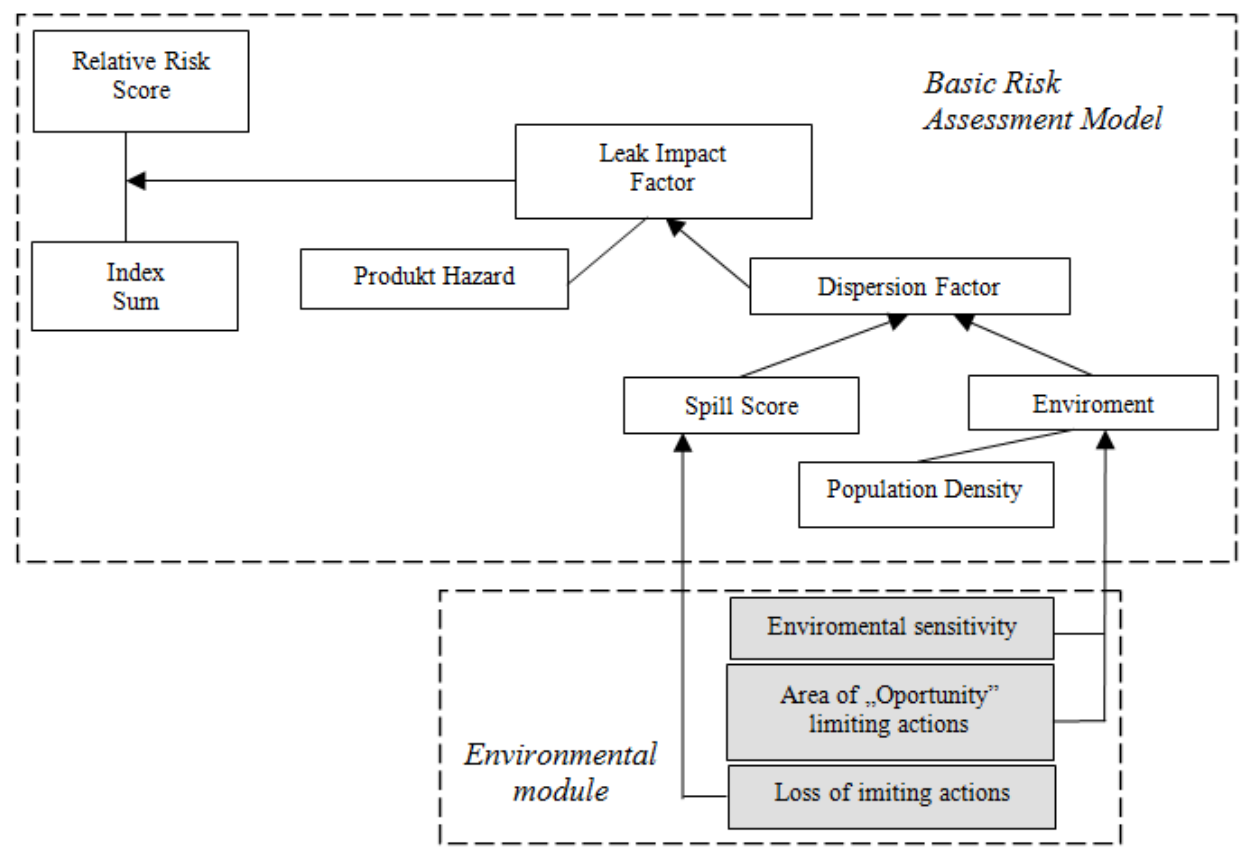

Fig.2 Diagram of the interactions of the environmental module on the basic risk assessment model $[4,5]$

The environmental module takes into account:

- Environmental sensitivity - specially protected areas such as bird nesting sites, freshwater marshes, eaches, etc.

- Areas of great value - from archaeological sites, historic sites to high land values;

- Leakage minimization measures - a set of tools and procedures that allow the operator to immediately reduce the leak.

In the risk assessment, the first two elements can increase the impact of the environment by a maximum of 1 point, while minimizing the effects of leaks can reduce the consequences of leakage by $50 \%$, resulting in a leakage increase but a maximum of 1 point.

\subsection{Damage Module}

The failure module is applicable when the expert evaluator sees the need to increase the participation in the risk assessment of the impact of the type of materials used, the mounting and operating stresses and the initiators of the damage. The basic evaluation method does not provide for extreme situations, such as periodic over-exposure of normal operating stresses or maximum operating pressure of a factor under rare load scenarios. Correction of the leakage rate for gas networks varies from -2 points to 0 , for liquid transport pipes the point reduction is $50 \%$.

\subsection{History of Leaks Module}

Based on statistical data. Understood as a higher probability of a failure on a pipeline that has been repeatedly leaked in the past. Only the nature of their causes is ignored by using the number of failures that is assessed in the baseline method. If, however, in a comparative analysis with other sections of the network for the fragment analyzed, the number of failures is much higher, the value of the factor can be as high as $10 \%$, if the average is $5 \%$ and if it does not deviate from the average for the entire network, i.e. unchanged. This coefficient corrects down the number of points awarded in the basic method indices.

\subsection{Stress Module}

All the factors characterizing the level of stress, employees can be divided into three categories. Depending on the category, the basic indicator is reduced. If you don't have sufficient information about stress, then the level of stress should correspond to the following:

- Very high stress -20 pts;

- Neutral 0 pts;

- Very low stress +20 pts. 


\subsection{Sabotage Module}

The threat of vandalism, sabotage or other intentional destruction of property is omitted in the basic assessment. Third party damage indicators are taken into account for unintentional actions. The primary purpose of the sabotage operation is to trigger the immediate effect of the sealing of the pipeline. Points are added for each preventive measure applied.

\section{The Adaptation of WKM's Risk Assessment Method into Risk Assessment Method for Heating Networks}

The method of assessing the risk of district heating networks is based on the WKM method, leaving the basic method and the group of modules used optionally. WKM method is a method of risk assessment addressed to gas networks and transmission pipelines, pipelines embedded in the ground or marine pipelines and can be used to evaluate the heating distribution network. Risk assessment district heating networks is designed for high parameter and low-parameter networks. The high-parameter networks are designed for a temperature range of $130 / 70^{\circ} \mathrm{C}$, and for nominal pressure of $1.6 \mathrm{MPa}$. The low-parameter networks are designed for a temperature of $95 / 70^{\circ} \mathrm{C}$ and nominal pressure of $0.6-1 \mathrm{MPa}$.

\subsection{Model of Risk Assessment}

The basis for explaining the adaptation changes in the basic risk assessment model is the characteristics of the district heating network. The modern district heating network is made of pre-insulated pipe technology, directly laid in a sand dirt or flyover to form an overhead network. $40 \%$ of district heating networks in Poland are still in the channel technology, which means that regardless of whether they are underground or terrestrial pipes, they are laid on steel sliding supports and covered with a thermal insulation sheath. In the case of the underground network, both pipes on the supports are closed in a channel made of prefabricated reinforced concrete. It is ventilated and filled with atmospheric air. In the case of nets on the air, pipes are also laid on sliding supports but insulated with a polyurethane foam and each is individually covered with a metal protective sheath. In the channel technology, there are fixed points for heating chambers in which control and measuring equipment, tees and shutoff valves are installed. In the case of pre-insulated tees, the valves are located in the ground in a heat insulating jacket. Pre-insulated technology is an automatic leak detection system. The control and measurement equipment is installed in heating nodes, i.e. at the points of heat reception.

In practice, the supply and return networks are twin. Therefore, in the base method, the principle that the rated section of the district heating network consists of two parallel running pipe lines, Power supply and return network. It is possible to conduct a risk assessment separately for the mains and the return network if the supply lines are different from the return lines in the range of at least one parameter, i.e. geometry, manufacturing technology, operating conditions, or the route during breakdown.

High-temperature district heating is a system of pipelines arranged in an area of intense urbanization, increased traffic, railways, tramway and rail. They are characterized by a strongly developed tree structure with the occurring closures of the network regions or rings. Direct neighborhood of other network media, the intensity of construction and repair work is the standard operating environment of the district heating network. In the process of adaptation of WKM method scores for core indicators remained unchanged.

\subsection{Third Party Damage Index}

The network is located in the strong area's urbanized characterized by the strong activity of the inhabitants. Compensation is the fact that the network is in detail and well-identified.

- Scale of evaluation 0-100pkt:

- The level of activity - the intensity of earthmoving, construction 0-30 pts;

- Active on-line system - a system of reporting irregularities noticed current from a third party - the residents $-0-20 \mathrm{pts}$;

- Marking and protection of the heating network 0-20 pts;

- State of the route - protection against the entry of third parties to heat exchangers sub stations, heat chambers, marked heating vent channels, marking the position of shut-off valves - 0- 10 points;

- The frequency of patrolling - cyclic control by the technicians receiving heat chambers and route network $0-20$ points, When the whole network and all substations are controlled at least once a week).

\subsection{Corrosion Index}

The corrosion index similar to the WKM risk assessment method consists of three components:

- Atmospheric corrosion 0-20 pts;

- Internal corrosion 0-20;

- Corrosion of the pipe under the ground. 0-60 pts. 
In the process of adaptation of the risk assessment method for heat distribution networks, point scales have been significantly altered leaving a total score of 100 points. Atmospheric corrosion 0-20 points (a network of pre-insulated always 20 points). - evaluation refers to the surface heat pipelines, but also below the surface carried out in the channels.Further scoring depends on the type of atmosphere (max. 10 pts), equipment occurring (max. 5 pts) and corrosion protection applied (max 5 pts).

Internal corrosion ( $0-40$ points) is caused by the interaction between the inner wall surface of the pipeline and flowing agent - water network. This corrosion can be caused by the content of oxygen and other substances which catalyze the corrosion process of the deposition of scale in the form of e-g. calcium and magnesium carbonate. The evaluation will assess the quality of the water network, manner of its treatment and physic-chemical parameters max. 25 points. Corrosion is evaluated as a function of class project and the $\mathrm{pH}$ of the water network (max. 5 pts.) and the used control system and prevention of corrosion (max. 10 pts.).

Factor in the corrosion of the pipe is replaced by a corrosion assessed in from the outside and that the limit of 0 40 pts.

The following are subject to evaluation;

- $\quad$ Pipe age (max 5 pts);

- $\quad$ Occurrence of inductive AC (max 5 pts);

- Insulation status ( $\max 20$ pts);

- $\quad$ Soil quality (max 5 pts);

- $\quad$ Pipe route (max $5 \mathrm{pts}$ )

\subsection{Design Index}

Design index consists of the following evaluation factors:

- $\quad$ Safety factor of the pipe (max. 30 pts.)

- Safety factor of network segment (max. 30 pts.)

- The possibility hammer (max. 10 pts.)

- Probability of earth movements (max. 10 pts).

\subsection{Incorrect Operations Index}

The index of incorrect operations consists of the following factors:

- Construction (max. 25 pts) - including detailed analysis of the process control, material selection, execution of welds and the assembly of the leak test;

- Operation (max. 50 pts) - assessment of employee training activities, routine activities and equipment for control and security systems;

- Maintenance (max. 25 pts) - including assessment of document management procedures used for repair schedule.

\subsection{Leak Impact Factor}

The leak impact factor has been completely changed to that proposed by the WKM. It consists of two factors: product hazard and distraction.

\section{Product hazard}

In the case of unsealing of the heat pipe, the hot water starts leaking as evaporates at ambient temperature and pressure. The product hazard is not permanent, but only a short-term process with no flammability, toxicity, or reactivity. A fixed value of 16 points was adopted, which corresponds to the maximum point value in the case of a permanent hazard of 10 points, while in case of short-term, it is mid-scale $0-12$ points, i.e. 6 points.

In the case of district heating networks, the hot water is always a factor in the outflow, so, there are no indicators for correcting the leakage due to the fact that the leakage agents are chemically different. The leakage result is the arithmetic mean of the leakage and permeability of the soil. In the worse case, it is 1 point and the most preferred 5 points.

Population density assessed as a function of density and destination of buildings on the territory of which the district heating network is used. WKM proposed four classes of population density, which are appropriately awarded from 1 to 4 points.

The model for the assessment of the district heating network risk in the basic and extended scope is presented in Figure 3. 


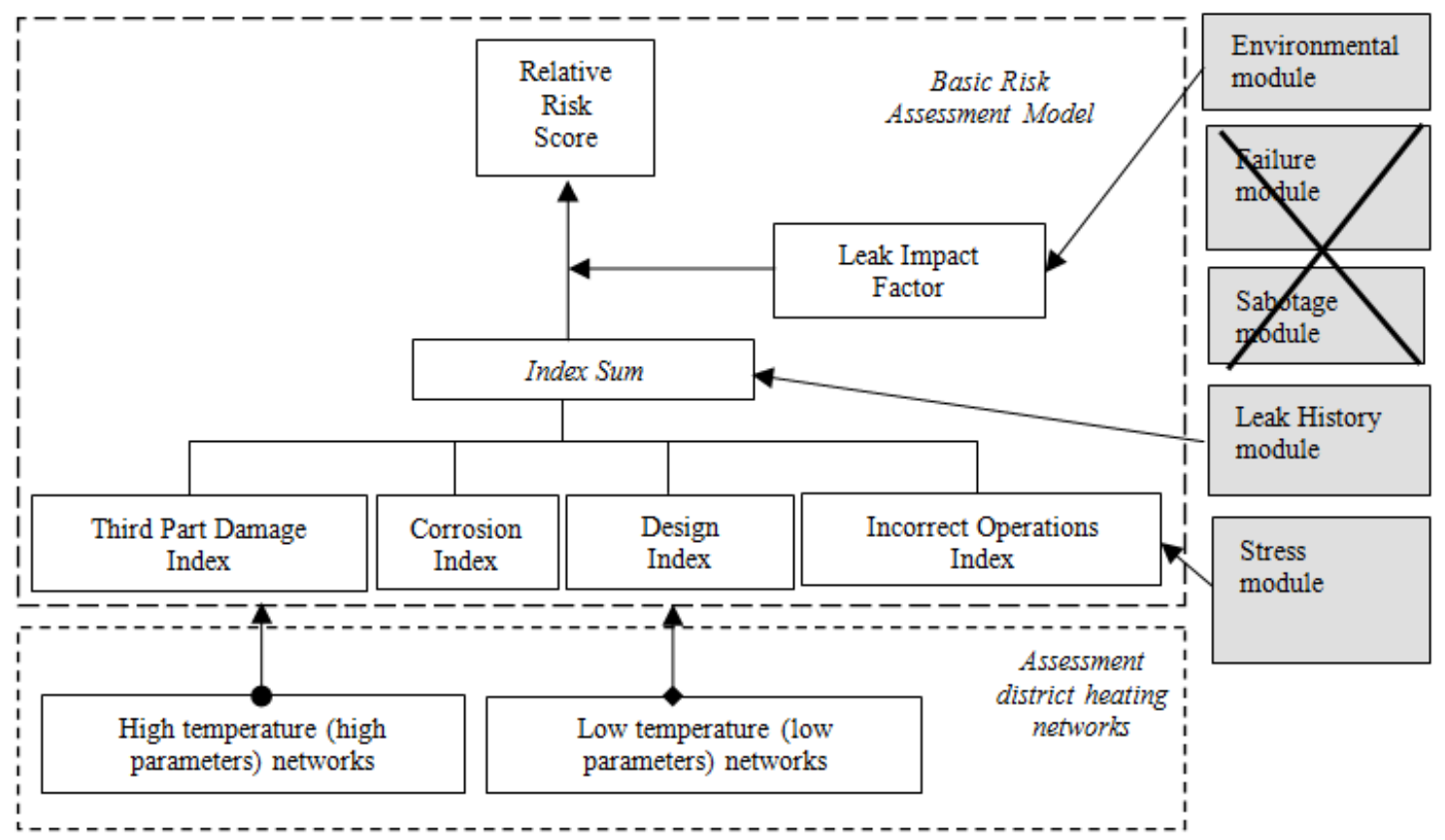

Figure 3.The model for the assessment of the district heating network risk in the basic and extended scope

Based on the above characterized model, a program was developed to build a relational database for district heating risk assessment.

\section{Relational Data Model Risk Assessment of District Heating Networks}

In mathematical terminology - the database is a collection of relations. Hence, historically derived relational data model and relational database. Representation of a relationship is a two-dimensional table composed of columns and rows.

The columns correspond to the factors that are evaluated by the score, the sums of which form the points of the indicator points, and the rows correspond to the sections of the district heating network that have been designated according to the assumptions adopted for the risk assessment. As already mentioned, the supply network is a twin network for the return network, so the rated district heating network segment is two wires with the same geometry and operating parameters. In the case of low-parameter networks, these may be three identical wires and in some cases even four. If they differ in dimensions, technology or operating parameters, then the relational basis for the risk assessment of the district heating network is created separately for each piping system.

The relational database has a fixed number of columns defined by the risk assessment method described above, while the number of rows is the result of network divisions on the sections to be evaluated.

\section{The structure of the relational database of risk assessment for the heat distribution network}

The risk assessment relational database consists of layers. Primary layer corresponds to four indexes: third party damage, corrosion, design and abnormal operations. Each index tab contains factors that are subject to evaluation. The evaluator does not use point scales. The rating is a selection of one of the suggested responses in the drop-down menu. For users, the selected responses are displayed, after validation, the program counts the points that are uniquely assigned.

The following figure 4 shows a detail of the algorithm for the evaluation the index of abnormal operations:Each table defines a so-called identifier. The master key which is the column containing the ID codes of the separated heating network sections. These codes correspond to sections of the heating network on the network graph that map the actual network structure $[7,8]$. 

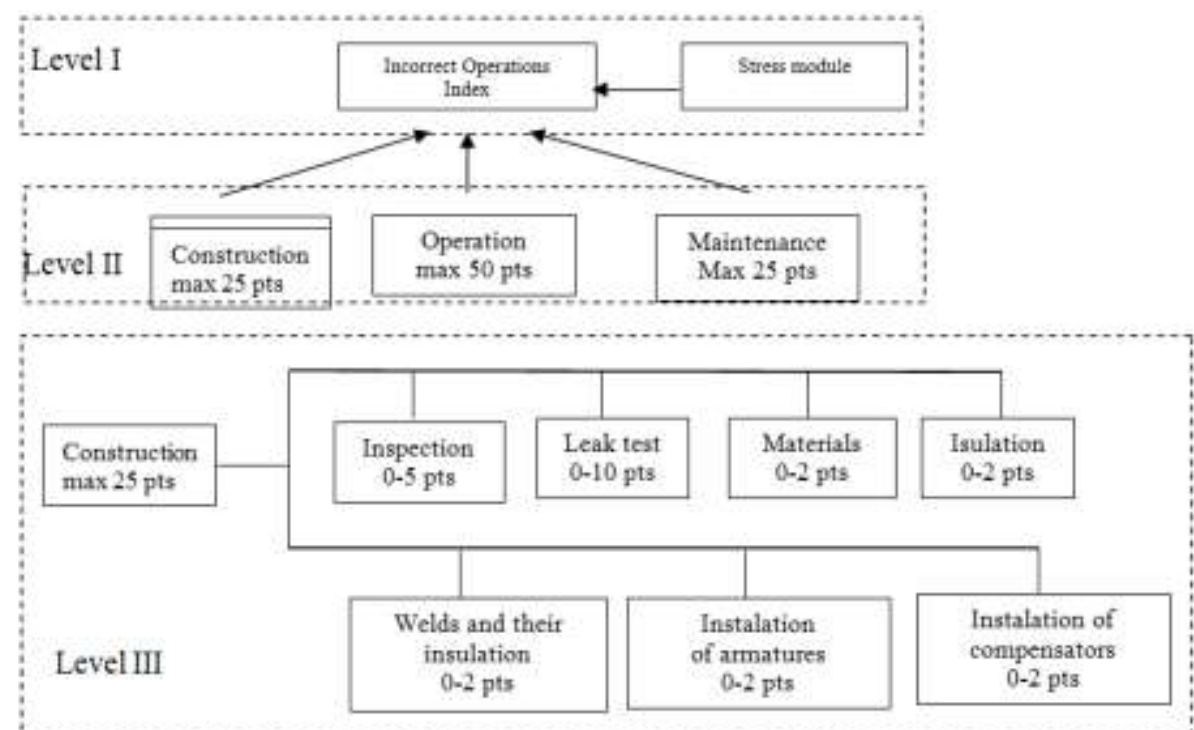

Fig. 4 The algorithm for the index evaluation of abnormal operations [6]

\section{Scoring algorithm}

Construction maximum 25 points.

Assessment are subject to the following elements:

a) inspection (supervision and control);

b) leakage test (pressure);

c) materials;

d) insulation;

e) welds and their insulation;

f) installation of compensators;

g) installation of armatures (valves).

Other indexes have a similar assessment structure. The result is a relational database of scores and allows you to assess the level of risk audited heating network.

Each rating of the network corresponds to a line where point values correspond to the level of risk. This is a relative rating that refers to the sections in the same district heating network. The result indicates which of the analyzed episodes is in the group at risk of failure.

Figure 5 shows a screenshot of the software program for risk assessment in the environment of the SimNet SSV Heat when entering data into the base in the environment module.

The selection of network sections, bordering the area of cover by security architecture in this case the city center is highlighted by yellow.

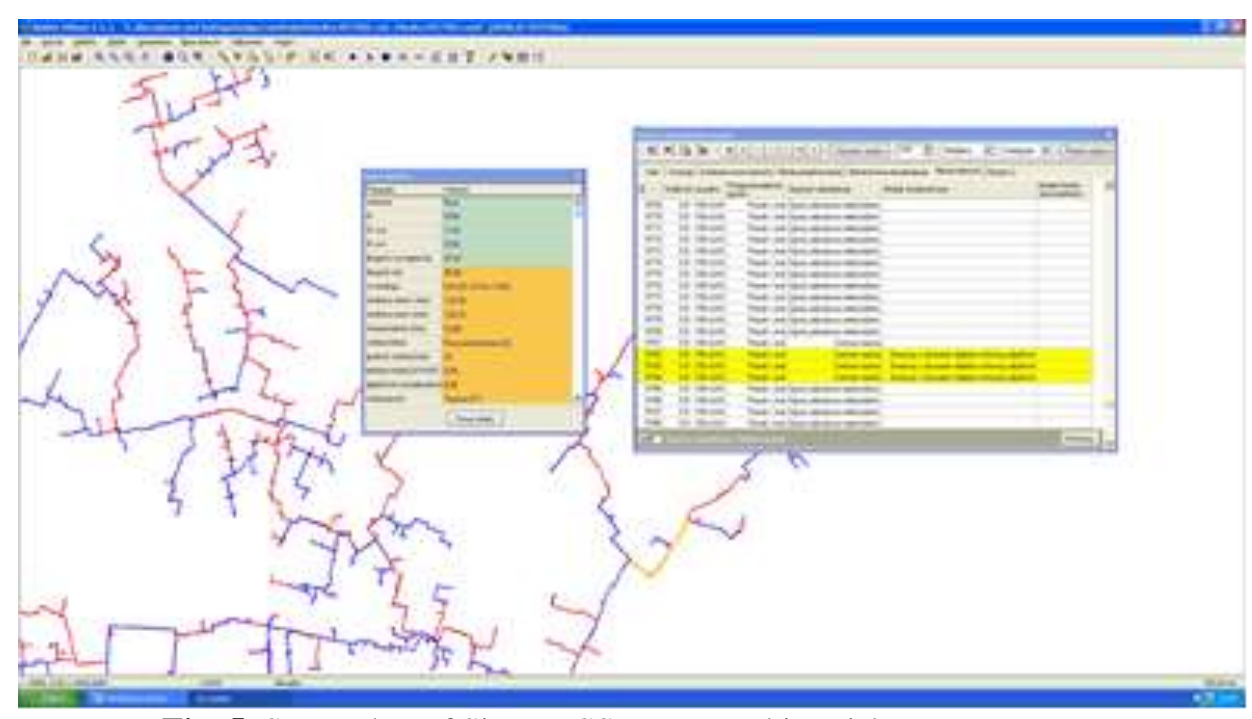

Fig. 5. Screenshot of SimNet SSV Heat making risk assessment [7] 


\section{Study Case}

High pressure heating network is in an urban area with a typical multi-family housing and an extensive industrial infrastructure forming industrial zone. The total length of the route network is about $70 \mathrm{~km}$ and is made of pre-insulated technology and channel entirely sunk under the ground is supplied from a source of heat to 780 heat exchangers substations.

Relational database risk assessment consists of 42 columns and 6229 rows. It means that 6229 separated network segments are made to assess the level of risk. Appointment of December dust can assign the same parameter for all sections (of arcs), which significantly speeds up the introduction of the source data into the database.

Relational database assessment of risks in heating networks created as a development program package SimNet S VV Heat is a program that simulates static heating network. This is a network computing applications of GIS, which means that utilize GIS technology to store and display the graphic (geographical) and alphanumeric information about objects district heating network. It can as well as associate information such as graphic. Figure section of the heating network on a digital map with its description in the database alphanumeric provides convenient connection for the user to modify objects in a graphical environment with automatic changes with regard to the descriptive database.

Construction of the base necessary for risk assessment based on the presented methods is timeconsuming because of the detailed description of each element of the heating network. With the progress of the operation heating network, the modernization works or investment program changes will be gradually introduced to the network infrastructure.

\section{Acknowledgement}

This research was supported by Fluid Systems Ltd. The input data has been consulted with the company.For over 20 years Fluid Systems Ltd. develops software for hydraulic modelling of fluid networks (heating, gas, pressurized sewerage, water). The company holds a good reputation in the field of simulation software and currently is a major supplier of simulation software packages for fluid networks on the Polish market. It also offers the software for static and dynamic simulation and optimization of selected parameters of fluid networks.

(www.fluidsystems.pl)

\section{Journal Pepers:}

\section{References}

[1]. A.Głuszek, Trasport pipeline, Transmission pipelines point method of relative risk assessment. Inspector- Bimonthly issued by the Office of Technical Safety, no. winter 2017, pp 9-13

[2]. A.J. Osiadacz, M. Chaczykowski, Ł.Kotyński, The software package for simulating static heating networks, Heating, Ventilation, no.12/2012, ISSN: 0137-3676, pp 526-529

[3]. J Szybka, Z., R. Pilch, Forecasting the failure of a thermal pipeline on the basis of risk assessment and exploitation analysis Maintenance and Reliability 4/2011; pp 5-10

Books:

[4]. W.K. Muhlbauer, Pipeline risk Management Manual, a tested and proven system to prevent loss and assess risk, second edition, Gulf Publishing Company, , 1996.

[5]. W.K. Mulhlabauer, Management of pipeline risk, Polish edition translated by A.J. Osiadacz, Fluid Systems Sp. z o.o., 2013

\section{Proceeding Papers:}

[6]. M. Kwestarz, Safety and risk in the operation of heating systems, X-th Technical Conference of the Polish District Heating Chamber of Commerce, Poland, September 2014r.

[7]. M. Kwestarz, Risk analysis in the operation of fluid networks on the example of heating network, Scientific Conference on Energy Efficiency in the Operation of Fluid Networks, State Higher Technical School, Poland, June2014

\section{Thesis:}

[8]. E.Stojak. Risk analysis of district heating network during exploitation time, Engineering thesis, State Higher Technical School, Ciechanow, Poland 2016 